\title{
Statins: in the beginning
}

\author{
${ }^{1} \mathrm{KS}$ Lyons; ${ }^{2} \mathrm{M}$ Harbinson \\ ${ }^{1}$ Research Fellow, Department of Therapeutics and Pharmacology, Queen's University, Belfast; ${ }^{2}$ Senior Lecturer, Department of Therapeutics \\ and Pharmacology, Queen's University, Belfast, and Consultant Cardiologist, Belfast Health and Social Care Trust, UK
}

ABSTRACT Since the first human trial of a hydroxymethylglutaryl-coenzyme A (HMG CoA) reductase inhibitor in 1978, the growth in importance of this drug class, both financially and medically, has been staggering. The aim of this paper is to summarise how this drug class was developed, highlighting the role of Akira Endo.

KEYWORDS Compactin, Akira Endo, lovastatin, statins

DECLARATION OF INTERESTS No conflict of interests declared.
Published online December 2009

Correspondence to KS Lyons, Department of Therapeutics and Pharmacology, Whitla Medical Building, 97 Lisburn Road, Belfast BT9 7BL

tel.+44 (0)2890 975772 e-mail klyons0I@qub.ac.uk
Hydroxymethylglutaryl-coenzyme A (HMG CoA) reductase inhibitors (statins) are now one of the most widely prescribed medications worldwide, with an estimated 2 million people in the $\mathrm{UK}^{\prime}$ and more than 18 million people in the USA currently taking a statin. ${ }^{2}$ From their development in the 1970s the impact of statins on the treatment of cardiovascular disease been staggering. Their story is an interesting portrait of modern-day therapeutics and highlights the complexity of the development process.

\section{THE DISCOVERY OF COMPACTIN}

The first statin was discovered in the 1970s by a research group working at Sankyo Pharmaceuticals in Tokyo. Over the previous decades, a steady accumulation of observational data such as the Framingham study had demonstrated a link between raised serum cholesterol and cardiovascular events. ${ }^{3}$ Therapies available at the time, such as bile sequestrants, were poorly tolerated and had limited efficacy so the focus changed to target effective cholesterol-lowering therapies aimed at blocking one of the many steps in the synthetic pathway of cholesterol from acetyl coenzyme A. ${ }^{4}$

The research team at Sankyo was the first to synthesise such a substance. They were headed by a young researcher, Akira Endo, who had returned to Sankyo after two years at the Albert Einstein College of Medicine in New York. Endo initially studied organic chemistry and applied microbiology at Tohoku's University College of Agriculture, and joined Sankyo after his graduation in 1957. ${ }^{5}$ During his time in the USA he had been surprised at the levels of coronary heart disease and hypercholesterolaemia in the population, which was something he had not experienced at home in Japan. He was aware of the work of the German chemist Konrad Bloch on cholesterol biosynthesis and became interested in the idea of developing a cholesterollowering drug. Endo had written to Bloch in 1965 to enquire about working in his laboratory, but at that time a postdoctoral position was not available. He then spent two years at the Albert Einstein College of Medicine working on cell wall biosynthetic enzymes. ${ }^{6}$

When Endo returned to Sankyo in 1968 he began work on the quest to find an effective cholesterol-lowering drug. He worked on the premise that some microbes might produce inhibitors of enzymes necessary for cholesterol synthesis as a means of defence. ${ }^{4}$ With his research team, he began to examine microbial culture broths previously screened for antibiotics, for evidence of an inhibition of cholesterol synthesis.

At that time the assay for HMG CoA activity was complicated and involved the incubation of microsomes with radio-labelled ${ }^{14} \mathrm{C}-\mathrm{HMG} \mathrm{CoA}$, followed by the extraction and separation of ${ }^{14} \mathrm{C}$-mevalonate. ${ }^{7}$ This process was too laborious and expensive to be carried out on a large number of samples. Instead, two separate processes were employed. The first step involved measuring the incorporation of ${ }^{14} \mathrm{C}$-acetate into sterols. Compounds that inhibited this process were then tested for the inhibition of sterol synthesis from ${ }^{3} \mathrm{H}$-mevalonate. If this step was not inhibited then it was concluded that the compound acted on the initial conversion of HMG CoA to mevalonate by HMG CoA reductase. ${ }^{5}$

After thousands of experiments over a two-year period the Sankyo team eventually isolated a molecule that appeared to be effective, ML236-B. ${ }^{8}$ The substance was derived from cultures of Penicillium citrinum, a mould isolated from the rice of a vendor in Kyoto, and purified by solvent extraction, chromatography and crystallisation. ${ }^{4}$ The molecule contained a portion with a chemical structure very similar to mevalonate, the product of the HMG CoA reductase reaction. This finding fitted well with the theory that it acted via competitive inhibition of the enzyme HMG CoA reductase.

The efficacy of the compound, which was named compactin, was quickly demonstrated in animal models, including hens, dogs and monkeys. The first tests in rat models had been unsuccessful, demonstrating an initial 
fall in cholesterol in the first few hours followed by a rapid return to normal levels. This was later found to be due to an unexpected increase in hepatic levels of HMG CoA reductase in rodents, which did not occur in other animal models. ${ }^{9}$ This finding had caused a team from Beecham Laboratories in England to cease development of compactin, which they had isolated from another Penicillium species around the same time. ${ }^{5}$ However, Endo's team persisted. Short-term studies in laying hens revealed impressive reductions in total cholesterol, which provided sufficient evidence for further testing in other animal models.

In 1977, an 18-year-old Japanese woman with familial hypercholesterolaemia became the first human to be treated with compactin. ${ }^{4}$ A year later Sankyo started the first phase I clinical trial of the drug, with a phase II trial following shortly after. ${ }^{10}$ The results were impressive, with total cholesterol lowering in the region of $30 \%$; however, in 1980, the development of the drug was stopped. The reason for this was never released officially but was thought to be due to the finding of an excess incidence of lymphomas in dogs treated with particularly high doses of $100-200 \mathrm{mg} / \mathrm{kg} / \mathrm{d}$ in long-term toxicity studies. ${ }^{5}$ Endo later noted that toxicity studies with other statins never used doses as high as those used for compactin. ${ }^{5}$

\section{DEVELOPMENT OF LOVASTATIN}

In 1966, Sankyo had provided Merck with samples of compactin and pharmacological data on the drug as part of a confidential agreement. Over a decade later, in 1978, after carrying out independent studies with compactin, Merck independently isolated a new HMG CoA reductase inhibitor, lovastatin (then called mevinolin), from an Aspergillus species. At much the same time, Endo, now working at Tokyo Noko University, had also isolated a compactin analogue with a slightly greater efficacy, which he called monacolin K." It soon became clear that monacolin $\mathrm{K}$ and lovastatin were the same compound. Because lovastatin had been discovered three months before monacolin $\mathrm{K}$, a patent for lovastatin was approved in the USA, as priority was given to the fact it had been discovered first, even though the patent application was made later. However, in many other countries, 'time of application' was given priority. By then the patent rights for monacolin $\mathrm{K}$ had been sold to Sankyo for $¥ 35$ million (equivalent today to approximately $£ 255,000$ ). ${ }^{6}$

Although the development of this drug was also halted in 1980 following the compactin toxicity study findings, some small-scale clinical investigations by US physicians and additional toxicity studies (which suggested that the 'lymphomas' were actually histological changes due to massive drug doses rather than malignancies ${ }^{8}$ ) led to Merck resuming the development of lovastatin, which was finally given US Food and Drug Administration approval in 1987, nine years after its discovery.
The fact that Sankyo held the patent to lovastatin outside the US was a driving factor in Merck searching for yet another HMG CoA reductase inhibitor. In 1988 a Merck team developed a more potent semi-synthetic derivative of lovastatin which differed only by an additional side-chain methyl group. This was named simvastatin and subsequently marketed worldwide.

In 1991 pravastatin, derived by a biotransformation of compactin, was developed by Sankyo. Four more statins followed: fluvastatin (1994), atorvastatin (1997), cerivastatin (1998) and, most recently, rosuvastatin (2003), all of which are synthetic products. Cerivastatin was eventually discontinued in 2001 following several deaths from rhabdomyolysis. ${ }^{9}$

As further evidence into the pharmacological effects of statins has become available, it is clear that their protective effects are due to more than simple modulation of lipoprotein metabolism. Beneficial effects on atherogenesis, inflammation and markers of endothelial function $^{12}$ have been demonstrated in various disease models, independent of low-density lipoprotein lowering, leading to studies investigating the effects of statins in conditions as varied as sepsis and chronic obstructive pulmonary disease. ${ }^{13,14}$ These effects, which could benefit a multitude of conditions beyond hypercholesterolaemia, could not have been predicted by Endo and his colleagues as they set out to find a simple enzyme inhibitor. Indeed, the complex interactions which come into play when targeting any metabolic pathway can prove crucial to the success of a therapeutic agent, and are more often a matter of luck than foresight.

\section{CONCLUSION}

The importance of the role played by Endo in the discovery of statins was highlighted when he was awarded the 2008 Lasker-DeBakey Clinical Medical Research Award. ${ }^{15}$ The effectiveness and impressive safety profile of statins have now been demonstrated by numerous randomised controlled trials since the publication of the landmark 4S study with simvastatin in $1994 .{ }^{16}$ Scotland, with its high incidence of hypercholesterolaemia, provided subjects for the first of the primary prevention trials (The West of Scotland Coronary Prevention Study), ${ }^{17}$ with more than 6,000 men recruited. This study is still producing results, with one of the longest periods of patient follow-up of any statin trial. ${ }^{18}$

The story of statin development will always be seen as a great success, both scientifically and financially, with statin prescriptions costing around $£ 0.6$ billion in the UK and more than $\$ 12$ billion in the US in 2005 and costs increasing annually. ${ }^{19}$ It illustrates how epidemiological data can provide the evidence for theories on disease processes, which can in turn lead to the development of targeted therapies to modify these 
processes. The statin story may have been rather different, however, had any of the problems which jeopardised the early development not been overcome by thorough and methodical experimentation.

\section{REFERENCES}

I Mayor S. NICE calls for wider use of statins. BMJ 2006;332:256. doi: I0.1 I36/bmj.332.7536.256-d

2 Woloshin S, Schwartz LM, Kerin K et al. Estimating the impact of adding $\mathrm{C}$-reactive protein as a criterion for lipid lowering treatment in the United States.J Gen Intern Med 2007;22:197-204. doi:10.1007/ sl 1606-006-0033-z

3 Steinberg D. The statins in preventive cardiology. N Engl J Med 2008; 359: 1 426-7. doi:10.1056/NEJMp0806479

4 Endo A.The discovery and development of HMG Co-A reductase inhibitors.J Lipid Res 1992; 33:1569-82.

5 Endo A. A gift from nature: the birth of the statins. Nat Med 2008; 14:1050-2. doi: $10.1038 / \mathrm{nm} 1008-1050$

6 Endo A. The origin of statins. Atheroscler Suppl 2004; 5:125-30. doi:10.1016/j.atherosclerosissup.2004.08.033

7 Goldfarb S, Pitot HC. Improved assay of 3-hydroxy-3-methylglutaryl coenzyme A reductase. J Lipid Res 197।; 12:5 |2-5.

8 Stossel TP. The discovery of statins. Cell 2008; 134:903-5. doi:10.1016/j.cell.2008.09.008

9 Tobert JA. Lovastatin and beyond: the history of HMG Co-A reductase inhibitors. Nat Rev Drug Discov 2003; 2:5I7-26. doi: I0.1038/nrdIII/2

10 Yamamoto A, Sudo H, Endo A. Therapeutic effects of ML-236B in primary hypercholesterolaemia. Atherosclerosis 1980; 35:259-66. doi:10.1016/002I-9I50(80)90I24-0
In the modern era, as drug companies become the driving force behind developments in therapeutics, the goals of financial benefit and health improvement have become inextricably linked. Statins are a fine example of a drug class that has achieved both.

II Endo A. Monacolin K, a new hypercholesterolemic agent produced by Monascus species. J Antibiot (Tokyo) 1979; 32:852-4.

12 Davignon J. Beneficial cardiovascular pleiotropic effects of statins. Circulation 2004; 109:39-43. doi:I0.I I6I/0I.CIR.0000I3I5I7.20I77.5a

I3 Falagas ME, Makris GC, Matthaiou DK et al. Statins for infection and sepsis: a systematic review of the clinical evidence. J Antimicrob Chemother 2008; 61:774-85. doi:10.1093/jac/dkn0I9

I4 Blamoun Al, Batty GN, DeBariVA et al. Statins may reduce episodes of exacerbation and the requirement for intubation in patients with COPD: evidence from a retrospective cohort study. Int J Clin Pract 2008; 62:I373-8. doi: I0.IIII/j.I742-I24I.2008.0173I.x

15 Thompson GR. Lasker award for the discoverer of statins. $\mathrm{Br} J$ Cardiol 2008; 15:294-5.

I6 Scandinavian Simvastatin Survival Study Group. Randomised trial of cholesterol lowering in 4444 patients with coronary heart disease: The Scandinavian Simvastatin Survival Study (4S). Lancet 1994; 344:1383-9.

17 The West of Scotland Coronary Prevention Study Group. Prevention of coronary heart diesease with pravastatin in men with hypercholesterolemia. N Engl J Med 1995; 333:I30I-7. doi:I0.1056/NEJMI995III6333200 I

18 The West of Scotland Coronary Prevention Study Group. Longterm follow-up of the West of Scotland Coronary Prevention Study. N Engl J Med 2007; 357:I477-86. doi:I0.I056/NEJMoa065994

19 Swanton RH.The National Service Framework: six years on. Heart 2006; 92:29I-2. doi:I0.II36/hrt.2005.066043

\section{HEALTHCARE-ASSOCIATED INFECTION Tuesday 2 February 2010 at the Royal College of Physicians of Edinburgh}

The internationally distinguished speakers assembled for this Hot Topic Symposium, organised by the Royal College of Physicians of Edinburgh, will address the issues of healthcare-associated infections as they affect medical and paramedical practitioners, and describe the current and likely future size of the problem along with possible solutions.

Topics include:

- Improving the quality of antimicrobial prescribing

- Clostridium difficile

- Infection control

All grades of medical, nursing and scientific staff and allied healthcare professionals are most welcome. Standard fee $\notin 75$, Nurses/AHPs/ Pharmacists $€ 45$.

Admission is free to medical students who register in advance.

In association with:

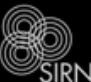

Full details of the programme and online registration are available from: http://events.rcpe.ac.uk

Or from:

Margaret Farquhar, Education, Training and Standards Department, Royal College of Physicians of Edinburgh, 9 Queen Street, Edinburgh EH2 IJQ Tel: + 44 (0) I3I 2473636 Email: m.farquhar@rcpe.ac.uk 